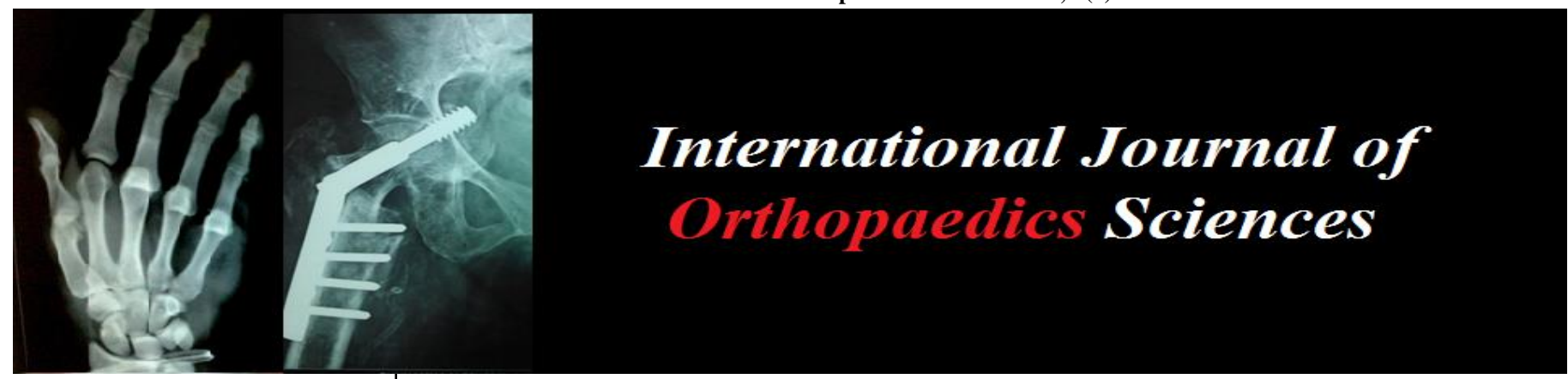

E-ISSN: 2395-1958

P-ISSN: 2706-6630

IJOS 2020; 6(3): 647-650

(C) 2020 IJOS

www.orthopaper.com

Received: 15-04-2020

Accepted: 18-05-2020

Sunil Shivanna

MBBS, MS (Orthopedics),

Fellowship in Arthroscopy and

Arthroplasty Designation: Senior

Resident, Department of

Orthopedics, Chamarajanagar

Institute of Medical Sciences,

Hamarajanagar, Karnataka,

India

Aarthi Thiagarajan

Department of Orthopedics,

Chamarajanagar Institute of

Medical Sciences,

Chamarajanagar, Karnataka,

India
Corresponding Author:

Sunil Shivanna

MBBS, MS (Orthopedics),

Fellowship in Arthroscopy and Arthroplasty Designation: Senior

Resident, Department of

Orthopedics, Chamarajanagar

Institute of Medical Sciences,

Hamarajanagar, Karnataka,

India

\section{Evaluation of outcomes of total knee replacement using forgotten joint score}

\section{Sunil Shivanna and Aarthi Thiagarajan}

DOI: https://doi.org/10.22271/ortho.2020.v6.i3j.2266

Abstract

Total knee arthroplasty (TKA) is one of the commonly performed procedure for osteoarthritis knee, rheumatoid arthritis, post traumatic arthritis etc. with a main goal of providing a pain free, stable and functional joint. Assessment of clinical outcomes should be done by both objective findings and by taking into account the patients point of view and his/her own evaluation of the functional trends of the treated joint. Forgotten Joint Score (FJS) is one such recently developed patient reported outcome measure (PRO), with better discriminatory power. The main aim of our study was to evaluate the patient reported outcomes in our patients who underwent TKA for osteoarthritis knee using FJS system and to analyse the temporal relationship of the outcomes of the time since surgery. Though there are various scoring systems, the "forgotten joint score" system is a disease specific and joint specific scoring system . We concluded that the best scores among our patients was obtained in patients operated 12 months and 24 months before the conduct of study. Therefore we can conclude that the maximum improvement in functional status of patients can be expected between 6months to 12 months after sugery. Age, gender had no affects on the outcomes and the FJS score. Patients with unilateral TKR as well as bilateral TKR both had equally better functional outcomes and FJS scores.

Keywords: Total knee arthroplasty, forgotten joint score, patient reported outcome

\section{Introduction}

Total knee arthroplasty (TKA) is one of the commonly performed procedure for osteoarthritis knee and other joint damaging conditions like rheumatoid arthritis, post traumatic arthritis etc. The main goals of performing a total knee arthroplasty is to provide a pain free, stable, functional joint. Hence evaluating the results of surgery is of fundamental importance for any orthopedic surgeon. Since the goal of orthopedic treatment is to reestablish functional status, it is important to assess clinical outcomes not only by objective findings but also by taking into account the patients point of view and his/her own evaluation of the functional trends of the treated joint ${ }^{[1]}$. Forgotten Joint Score ( FJS ) is one such recently developed patient reported outcome measure (PRO), with better discriminatory power and less ceiling effects compared to other traditional questionnaires like Knee injury and osteoarthritis outcome score (KOOS), Western Ontario and Mcmaster universities Osteoarthritis Index (WOMAC) , and Oxford knee score ${ }^{[2]}$. Moreover FJS is a disease specific as well as a joint specific scoring system which helps us to better evaluate the surgical outcomes of TKA. The aims and objectives of our study was to evaluate the patient reported outcomes in our patients who underwent TKA for osteoarthritis knee using FJS system and to analyse the temporal relationship of the outcomes of the time since surgery. We have also tried to evaluate the influence of factors like age, sex, unilateral and bilateral TKA on the FJS scores ${ }^{[1]}$.

\section{Materials and Methods}

A retrospective study was conducted considering 90 patients who were operated with TKA for primary osteoarthritis knee between may 2017 to October 2019 at our centre. These 90 patients were divided into three groups. The first set of patients were operated with TKA 24 months prior to study, the second set of patients were operated 12 months before study and the third set consisted of patients who were operated 6 months prior to study. The contact details of all the patients were collected and each patient were individually evaluated on follow up and the 
answers for FJS questionnaire was obtained. Following that the FJS score for each patient was calculated and the mean FJS was obtained for each group. These mean FJS of each group were statistically analysed so as to draw a conclusion regarding the temporal relationship of FJS with the patient reported outcomes post TKR. Patients operated with Primary TKR for osteoarthritis knee were only included and other complex primary TKA and revision TKA were excluded.
Results

\section{Difference between groups}

The FJS values for each group are non-normal, and hence the values are represented using median and interquartile range. The significant difference between the average FJS for groups are studied using Kruskal Wallis test at 5\% level of significance

Table 1: Difference between groups of patients in relation to time since surgery

\begin{tabular}{|c|c|c|c|c|}
\hline Group & $\mathbf{N}$ & Median (interquartile range) & Kruskal Wallis test value & p-value \\
\hline $\begin{array}{c}\text { Group 1 } \\
\text { Surgery before 24 months }\end{array}$ & 32 & $82.48(77.28,86.37)$ & & \\
\cline { 1 - 2 } $\begin{array}{c}\text { Group 2 } \\
\text { Surgery before 12 months }\end{array}$ & 28 & $79.55(72.73,87.22)$ & \multirow{2}{*}{39.55} & $<0.001$ \\
\cline { 1 - 2 } $\begin{array}{c}\text { Group 3 } \\
\text { Surgery before 6 months }\end{array}$ & 30 & $55.28(26.33,68.75)$ & & \\
\hline
\end{tabular}

Since $\mathrm{p}$ value is $<0.001$ it can be concluded that the average FJS value is significantly different for each group. Using Bonferroni test it can be seen that there is no significant difference between group 1 and 2 (surgery before 24 months and surgery before 12 months $(p>0.05)$. There is significant difference between the average FJS values between group 1 and 3 (surgery before 24 months and before 6 months) with $p<0.001$ as well as groups 2 and 3 (surgery before 12 months and before 6 months) with $p<0.001$.

\section{Difference between gender}

The FJS values for both males and females are non-normal and hence the values are represented using median and interquartile range. To compare the significant difference between the average FJS values among males and females Mann Whitney U test is used.

Table 2: Gender distribution of patients

\begin{tabular}{|c|c|c|c|c|}
\hline Group & $\mathbf{N}$ & Median (interquartile range) & Mann Whitney test value & p-value \\
\hline Male & 29 & $79.55(63.75,85.24)$ & -0.631 & 0.528 \\
\hline Female & 61 & $75 \quad 53.75,85.11)$ & & \\
\hline
\end{tabular}

From Table 2 it can be seen that there is no significant difference in the average FJS values between male and female since $p>0.05$

\section{Difference between age groups}

The FJS values for different age groups are non-normal. The values are represented using median and interquartile range. The significant difference in average FJS values between age groups is checked using Kruskal Wallis test.

Table 3: Age wise distribution of patients

\begin{tabular}{|c|c|c|c|c|}
\hline Age Group & $\mathbf{N}$ & Median (interquartile range) & Kruskal Wallis test value & p-value \\
\hline Up to 50 & 7 & $81.82(36.11,83.33)$ & & \\
\cline { 1 - 2 } $51-60$ & 33 & $77.28(66.66,86.37)$ & & \multirow{2}{*}{0.439} \\
\cline { 1 - 2 } $61-70$ & 43 & $77.28(55.0,83.40)$ & & \\
\hline More than 70 & 7 & $77.2875 .0,86.11)$ & & \\
\hline
\end{tabular}

From the above table it can be concluded that there is no significant difference in average FJS value among different age groups. Since $p>0.05$.

\section{Difference between patients with bilateral surgery and unilateral surgery}

The FJS values for patients with bilateral and unilateral surgeries are non-normal. Hence the values are represented using median and interquartile range. The significant difference in average FJS values among these groups are studied using Mann Whitney U test.

Table 4: Difference in outcome between bilateral TKR and unilateral TKR patients

\begin{tabular}{|c|c|c|c|c|}
\hline Type & $\mathbf{N}$ & Median (interquartile range) & Mann Whitney test value & p-value \\
\hline Unilateral & 44 & $77.28(58.75,86.31)$ & -0.065 & 0.948 \\
\hline Bilateral & 46 & $77.28(64.37,84.1)$ & & \\
\hline
\end{tabular}

The above table shows that there is no significant difference in the average difference among the patients with bilateral surgery and unilateral surgery since $p>0.05$

\section{Discussion}

In the present study we tried to evaluate the temporal relationship between the duration after total knee replacement for osteoarthritis knee with the patient reported outcomes measured using Forgotten joint score system. Apart from that we also tried to evaluate the effect of age, gender and unilateral total knee replacement versus bilateral total knee replacement on the FJS scores of the patient. 
With respect to temporal relationship, our study showed that the average FJS score for the patients operated before two years was 82.48 , it was 79.55 for patients who were operated before one year and 55.28 for patients who were operated six months before study.

In a similar study by Carlson et al. ${ }^{[3]}$, the average FJS scores for patients operated 6,12 and 24 months before the study was $59.4,72.5$ and 76.4 respectively. This is similar to the findings in our study. This is also similar to studies by Kim et al. ${ }^{[4]}$ and Thienpont et al. ${ }^{[5]}$ which had average FJS scores between 57 to 73.2 between qne and two years after TKR.

According to study by Carlson et al ${ }^{[2]}$, the maximum improvement after TKR is achieved between one to six months post surgery. The peak values in outcomes are reached at 2 years post surgery after which the improvement graph somewhat begins to flatten. Even in studies by Hiyaman et al. ${ }^{[6]}$ shows that best outcomes after TKR can be expected between one to six months post surgery and Merk Vincent et al. ${ }^{[7]}$ showed plateau in improvements after TKR reached after two years post surgery.

In our study we could find out that there is a statistically significant improvement in the FJS scores between six months and twelve months post TKR with a $p$ value $<0.001$ and we found no statistically significant improvement in FJS scores between 12 months and 24 months after surgery.

\section{Influence of Gender on TKR Outcomes}

In our study consisting of 90 TKRs, 29 were male and 61 were female we dint find any statistically significant differences in their post operative outcomes evaluated through FJS system with a $\mathrm{p}$ value of 0.528 .

In a similar study by Behrend et al. ${ }^{[8]}$, which evaluated 332 females and 208 males using FJS system, concluded that men has better post operative outcomes compared to women with a $\mathrm{p}$ value <0.01. Even a similar study by Linn $\mathrm{J}$ B et al. ${ }^{[9]}$ showed men had better results compared to women.

But other similar study by Theinpont et al. ${ }^{[5]}$ had concluded that gender has no effect on the patient reported outcomes post TKR. This is in line with the conclusions in our study.

\section{Influence of Age on TKR Outcomes}

In our study, there were 7 patients under 50 years of age, 33 were between 51 to 60 years, 43 were between 61 to 70 years and 7 were over 70 years of age. The average FJS scores for the above mentioned grouping is 81.82, 77.28, 77.28 and 77.28 respectively and we dint find any statistically significant difference in their FJS values with a $p$ value of 0.932. So age had no effect on outcomes post TKR. This is in line with other studies by Behrend et al. ${ }^{[8]}$ and Thienpont $e t$ al. ${ }^{[5]}$.

\section{Effect of Unilateral Versus Bilateral Tkr on the Post Operative Outcomes}

In a study by Latifi et al. ${ }^{[10]}$ evaluating the FJS between unilateral and bilateral TKRs had mean scores as 57.5 and 59.9 respectively with no statistical significance between their scores. Similarly study by Zeni and Syndel mackler et al. ${ }^{[11]}$ showed no difference in outcomes between unilateral and bilateral TKRs. Where as in a study by Bagsby et al. [12], Bilateral total knee replacement had good outcomes compared to unilateral TKRs.

In our study the average FJS score for both unilateral and bilateral cases was 77.28 and showed no difference in either of the patients.

\section{Conclusion}

Patient reported outcomes measures have become the best way to evaluate the functional status of the knee joint post TKR surgery. Though there are various scoring systems, the "forgotten joint score" system is a disease specific and joint specific scoring system with high validity and internal consistency.

Using forgotten joint scoring system in our study we concluded that the best scores among our patients was obtained in patients operated 12 months and 24 months before the conduct of study. Therefore we can conclude that the maximum improvement in functional status of patients can be expected between 6 months to 12 months after sugery.

We also conclude that age, gender had no affects on the outcomes and the FJS score, though few other studies states that with increasing age, the score will be better as the activity level and expectations of the elderly population goes down. Lastly patients with unilateral TKR as well as bilateral TKR both had equally better functional outcomes and FJS scores.

\section{References}

1. Behrend H, Giesinger K, Giesinger JM, Kuster MS. The "forgotten joint" as the ultimate goal in joint arthroplasty: validation of a new patient-reported outcome measure $\mathbf{J}$ Arthroplasty. 2012; 27(3):430-436.e1.

doi: 10.1016/j.arth.2011.06.035. Epub 2011 Oct 13

2. Giesinger K, Hamilton DF, Jost B, Holzner B, Giesinger JM. Comparative responsiveness of outcome measures for total knee arthroplasty. Osteoarthritis Cartilage. 2014; 22(2):184-9. doi: 10.1016/j.joca.2013.11.001. Epub 2013 Nov 18.

3. Carlson VR, Post ZD, Orozco FR, Davis DM, Lutz RW, Ong AC. When Does the Knee Feel Normal Again: A Cross-Sectional Study Assessing the Forgotten Joint Score in Patients After Total Knee Arthroplasty. J Arthroplasty. 2018; 33(3):700-703.

doi: 10.1016/j.arth.2017.09.063. Epub 2017 Oct 7

4. Kim MS, Koh IJ, Choi YJ, Lee JY, In Y. Differences in Patient-Reported Outcomes Between Unicompartmental and Total Knee Arthroplasties: A Propensity ScoreMatched Analysis. J Arthroplasty. 201732(5):1453-1459. doi: 10.1016/j.arth.2016.11.034. Epub 2016 Nov 27.

5. Thienpont E, Opsomer G, Koninckx A, Houssiau F. Joint awareness in different types of knee arthroplasty evaluated with the Forgotten Joint score. J Arthroplasty. 2014; 29(1):48-51. doi: 10.1016/j.arth.2013.04.024. Epub 2013 May 18.

6. Hiyama $\mathrm{Y}$ et al. Joint awareness after total knee arthroplasty is affected by pain and quadriceps strength. Orthop Traumatol Surg Res 2016, http://dx.doi.org/10.1016/j.otsr.2016.02.007

7. Merle-Vincent F, Couris CM, Schott AM, Conrozier T, Piperno M, Mathieu P, Vignon E. Osteoarthritis Section of the French Society for Rheumatology. Factors predicting patient satisfaction 2 years after total knee arthroplasty for osteoarthritis. Joint Bone Spine. 2011; 78(4):383-6. doi: 10.1016/j.jbspin.2010.11.013. Epub 2010 Dec 31.

8. Behrend H, Zdravkovic V, Giesinger J, Giesinger K. Factors predicting the forgotten joint score after total knee arthroplasty, J Arthroplasty. 2016; 31(9):1927-32. doi: 10.1016/j.arth.2016.02.035. Epub 2016 Feb 27.

9. Lim JB, Chi CH, Lo LE, Lo WT, Chia SL, Yeo SJ et al. Gender difference in outcome after total knee replacement. J Orthop Surg (Hong Kong). 2015; 
23(2):194-7.

10. Latifi R, Thomsen MG, Kallemose $\mathrm{T}$, Husted $\mathrm{H}$, Troelsen A. Knee awareness and functionality after simultaneous bilateral vs unilateral total knee arthroplasty. World J Orthop. 2016; 7(3):195-201. doi: 10.5312/wjo.v7.i3.195. PMID: 27004168; PMCID: PMC4794539.

11. Zeni JA, Snyder-Mackler L. Clinical outcomes after simultaneous bilateral total knee arthroplasty: comparison to unilateral total knee arthroplasty and healthy controls. J Arthroplasty. 2010; 25:541-546.

12. Bagsby D, Pierson JL. Functional outcomes of simultaneous bilateral versus unilateral total knee arthroplasty. Orthopedics. 2015; 38:e43-e47. 